\title{
The King-Nest
}

1. d4 d5 2. c4 e6 3. Nc3 dxc4 4. e3 b6 5. Bxc4 Nf6 6. Nf3 g6 7. e4 Bb7 8. e5 Ng8 9. 0-0 Ne7 10. Bg5 Bg7 11. d5 exd5 12. Nxd5 Bxd5 13. Bxe7 Qxe7 14. Qxd5 c6 15. Bb5 0-0 16. Bxc6 Nxc6 17. Qxc6 Rac8 18. Qe4 Bh6 19. Rad1 Rc7 20. Nd4 Rfc8 21. f4 Qh4 22. Qf3 Bf8 23. Nb5 Rc2 24. Nxa7 Bc5+25. Kh1 Rc7 26. Nb5 Rc8 27. Qb3 Bf8 28. Nd4 R2c4 29. Qxb6 Bc5 30. Qa6 Bxd4 31. Rxd4 R4c6 32. Qe2 Rc2 33. Rd2 Rxd2 34. Qxd2 Rd8 35. Qa5 Rd7 36. g3 Qd8 37. Qxd8+ Rxd8 38. a3 Rd7 39. Rc1 Rd8 40. b4 Re8 41. a4 Rd8 42. b5 h6 43. b6 Rb8 44. a5 Ra8 45. b7 Rxa5 46. b8Q+ Kg7 47. e6 Ra2 48. exf7 Kxf7 49. Qc7+ Kf8 50. Qd6+ Kg8 51. Rc8+ Kf7 52. Rf8+ Kg7 53. Qe7+ 1-0

\section{Echec 1.9-BB}

1. e4 e5 2. Nf3 Nc6 3. c3 Nf6 4. d4 exd4 5. e5 Ng4 6. cxd4 Bb4+ 7. Nc3 0-0 8. h3 Nh6 9. Bd3 d6 10. Bxh6 gxh6 11. Qc2 f5 12. Bc4+ d5 13. Bb5 Bd7 14. Bxc6 bxc6 15. 0-0-0 Be7 16. g3 Rb8 17. Qe2 Bc8 18. Kc2 f4 19. g4 Rb6 20. Rhe1 Ba6 21. Qd2 Qe8 22. Kc1 Qg6 23. e6 Qg7 24. Qc2 Qg6 25. Qxg6+ hxg6 26. Ne5 Bh4 27. Nxg6 Bxf2 28. Nxf8 Kxf8 29. e7+ Ke8 30. Re5 Rb4 31. Re6 Rc4 32. Kb1 Bxd4 33. Nxd5 cxd5 34. Rxa6 Kxe7 35. b3 Rb4 36. Kc2 c5 37. Rxa7+ Kf6 38. Ra6+ Ke5 39. Rxh6 Rb7 40. Kd3 Rb4 41. Rh5+ Ke6 42. Rf5 Be5 43. Re1 Rd4+ 44. Ke2 Re4+ 45. Kf1 Re3 46. Rxe3 fxe3 47. Ke2 d4 48. Kd3 Kd6 49. h4 Kd5 50. h5 e2 51. Kd2 d3 52. h6 Ke4 53. Rxe5+ Kxe5 54. h7 Kf4 55. h8Q 1-0

\section{CHESS COMPUTERS IN NOVI SAD}

\author{
Jan Louwman \\ Rotterdam, The Netherlands
}

During the great chess Olympiad in Novi Sad, Yugoslavia, four chess microcomputers were to be found in an open group of 218 chess-players. Those four non-human players achieved an exceptional tournament performance. In the unique ambiance, with all the stars of the chess firmament and thousands of visitors, the computers distinguished themselves by their fine quality of play.

\section{The Setting}

Church bells ring out over Novi Sad ... Try to imagine a sports complex covering 35 acres, because that is the size of the hosting accommodation. There is a number of vast halls, three of which are devoted to chess. One for National Men's teams, one for National Women's teams and the third for an Open Group. In the latter were 218 players, competing according to the Swiss system. Amongst this group were 4 computers, each of which also had to go the full nine rounds. Before the event some pessimism was evident, there were, after all, 3 IGMs, 32 IMs and 68 FMs. The Mephisto 68030 and the Final Chess Risc Card succeeded in vanquishing not a few of them, in games of considerable quality. Many of the strong players were Russian, playing in the hope of winning what for them were large prizes between $\$ 300$ and $\$ 1500$. They were originally-pleased to find themselves offered a computer to beat, an easy task they thought. But, that was not the way it was to be; often those amiable East Europeans would find themselves struggling for up to 8 hours or more just to finish a game and then lose as well. The Mephisto 68030 and the Risc Card with Ed Schröder's program, in particular, were often a match for even these strong players. (Risc means Reduced Instruction Set Computer; it is a chip which is particularly suitable for chess programs and the card, developed in Holland, runs at $16 \mathrm{MHz}$.)

\section{Impressed}

Using its fast components the Risc Card can evaluate 4000 positions a second. Considering how much chess knowledge is packed into Schröder's program, and what a brake it can be on the performance, we note that 4000 positions per second is very fast. This new configuration for the PC, and probably for other home computers in the near future, is a clear addition to our armament. Below, a game is presented played by Ed Schröder's new program. From the game you may conclude that the computers have been little impressed by their illustrious opponents. 
In the West we may find chess computers everyday things, but East Europeans find them something else. They crowded round, in front and even behind the machines. It got so busy we could hardly move, even to escape to heed the call of nature. The call was a flood of questions threatening to swamp me, posed in languages beyond my ken, or at least ones I failed to understand. Nevertheless they persisted with broken English and gesticulation in their attempts, still not always completely successful, to communicate. Yet, we had great fun. There is one story worth to be told. An African spectator became quite angry when I made a move for the computer. He replaced, to the astonishment of the human player at least, the piece, restrained me physically and then proceeded to make a different move. He failed to understand entirely what happens in such competitions. He made it very plain to me that I should clear off, so it was with considerable difficulty that we finally persuaded him that nothing untoward had taken place with the computer's move. When he finally grasped this he began to grin broadly and, as a gesture of friendship, landed me a clap on the shoulder I was to feel for days.

The Risc Card's play impressed us greatly, including the chess masters when they carried out the analyses after the event. The Mephisto 68030 impressed by its relentless ability to punish mercilessly the slightest mistakes of its frail and fallible human opponents. The Mephisto Polgar $10 \mathrm{MHz}$ also scored highly and even the Mephisto MM5, at $18 \mathrm{MHz}$, did well when it worked. It lost badly at one point when a good position was sacrificed by a breakdown that could not be repaired on the spot.

The Tournament Performance Ratings (TPRs) of all the computers are high, and deservedly so. The 68030 with a rating of 2360 was an exception, principally due to the way the Swiss system rewards a little bit of extra luck. Nevertheless it still justly deserved to be the leading computer. Grandmaster Tal, playing a 10-game speedchess match against the 68030, could only achieve a score of 5-5. Novi Sad was a tiring but enjoyable experience. With all the top players the computers were still a point of discussion. The keen-eyed reader will have spotted that three of the four programs were from the hand of Ed Schröder, a better compliment we can not give this Dutchman. Finally, the TPRs after 9 games for each machine were: Mephisto 68030 - 2360; Final Chess Risc Card - 2277; Mephisto Polgar $10 \mathrm{MHz}$ - 2249; Mephisto MM $518 \mathrm{MHz}$ - 2157. These ratings are FIDE standard.

It was with regret that we took our leave of the biggest chess circus ever seen. The computers, of course, knew nothing of this; they were not even aware of their incredible performance. The visitors, on the other hand, were all too aware of what they had seen those machines do.

\section{Final Chess Risc Card - Yusrei Mohamed (ELO 2210) \\ Novi Sad, 1990}

1. e4 e6 2. d4 d5 3. Nd2 c5 4. exd5 exd5 5. Bb5+ Bd7 6. Qe2+ Qe7 7. Bxd7+ Nxd7 8. dxc5 Nxc5 9. Nb3 0-0-0 10. Be3 Qc7 11. Nxc5 Bxc5 12. Qg4+ Kb8 13. Bf4 Bd6 14. Bxd6 Qxd6 15. Qxg7 Qe7+ 16. Kf1 Nf6 17. Re1 Qd6 18. Nf3 Rhg8 19. Qxf7 Ne4 20. h4 Rdf8 21. Qxh7 Nxf2 22. Kxf2 Qg3+ 23. Ke2 Qxg2+ 24. Kd1 Qxf3+ 25. Kc1 Rg2 26. Kb1 Rc8 27. Rhf1 Qg4 28. Re7 Qb4 29. a3 Qb5 30. Qd3 Qc6 31. Re2 Rg4 32. h5 Rg5 33. Qh7 Qb5 34. Ref2 a5 35. h6 Ka7 36. Qe7 Rh5 37. Qg7 Rc6 38. h7 Rch6 39. Rf8 Rxh7 40. Qg1+ b6 41. Qg8 Rc7 42. Ra8+ (announces within 4 seconds checkmate in 9 moves) Kb7 43. Qb8+ Kc6 44. Rf6+ Kc5 45. Qxc7+ Kd4 46. Qc3+ Ke4 47. Qf3+ Kd4 48. Qf2+ Ke4 49. Qf4++ 1 - 0

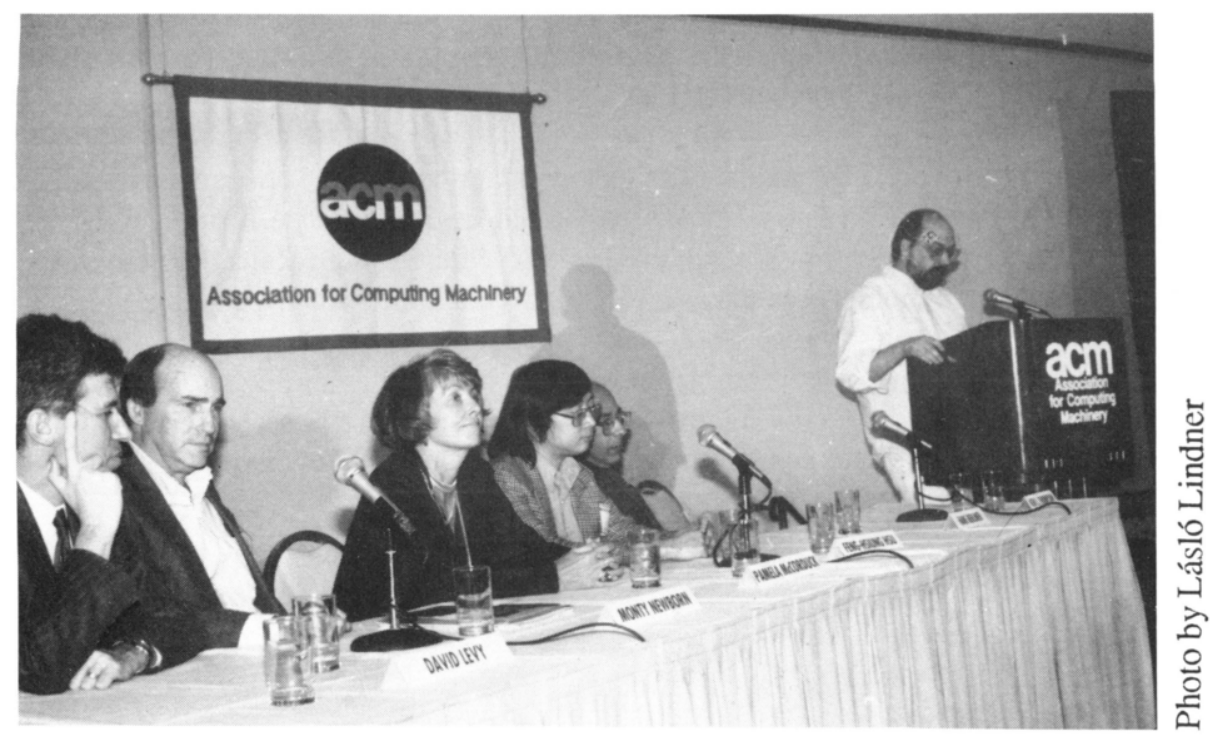

ACM's PANELLISTS, New York, November 1990. 